\title{
Repetitive stimulation abnormalities in 'epidemic neuromyasthenia': identification and implications (Abstract)
}

\author{
D. M. CURRIE \\ M.D.
}

\author{
A. SHELOKOV \\ M.D.
}

Four convalescent patients with suspected 'epidemic neuromyasthenia' (ENM) from the Ridgmar district of Fort Worth, Texas, were studied electrodiagnostically 6 to 7 months after the onset of symptoms. Although all patients still complained of subjective weakness and fatigability, no objective weakness was noted. Electromyography of axial and limb muscles and nerve conduction studies of several peripheral nerves were normal. Repetitive stimulation studies of deltoid, thenar, and hypothenar muscles were performed. In three of the four patients,, . one or two of these muscles showed late post-r exercise exhaustion (at $10 \mathrm{~min}$ after 'activation' byos maximal isometric exercise or tetany). These findings, if confirmed, imply that the weakness and fatigability so prominent in ENM may be due to dys के function of the myoneural junction.

( $A$ full report of these observations will be published elsewhere.)

\section{Discussion}

DR A. T. Richardson: I am becoming more and more impressed with the possibility that the disease we suffered at the Royal Free Hospital in 1955 may not correspond to that being described in some of the papers today. Dr N. Compston this morning described the clinical features of the Royal Free outbreak, notably the lymphoreticular and nervous system involvement. As has been said, a characteristic of the latter was the peculiar nature of the motor weakness which was detected clinically as a jerking on volitional effort and electromyographically as a grouping of motor unit potentials, this was particularly obvious during recovery from severe weakness but it did occur in mild weakness.

This phenomenon does not seem to have been observed in 'epidemic neuromyasthenia' as now described. I find it difficult to believe that if present it would have escaped detection on clinical examination or that Dr Donald Currie would have failed to pick up the groupings electromyographically had they been present in the cases at Fort Worth.

Professor A. Shelokov: He told me that he looked for them.

DR RICHARDSON: It is reasonable to equate on clinical grounds the 'Royal Free disease' (once called 'Akureyr disease' and later 'benign myalgic encephalomyelitis' surely the best term) with the cases at Addingtom Hospital, Durban, at Coventry Hospital and possiblop at the Middlesex Hospital, not least on the evidence of the clinicians involved in the three other epidemics wh $\vec{E}$ saw the Royal Free cases.

Further, it is possible to match the Royal Free and Coventry outbreaks on the basis of the electromyoe graphic (EMG) findings, it being that those shown to me by the late Dr John Schulman of Coventry matche 9 my own.

The use of repetitive stimulation in the EMG studies at Fort Worth brings in another parameter. It is unfortunate that that technique was not in general use i? 1955 and was not applied to the Royal Free cases. While I do not want to get into the subtleties of EMGs I mus? emphasize that while abnormal results of repetitive nerves stimulation seem to have been obtained at Fort Wort? they do not necessarily indicate a primary neuro-muscular defect. To give one example, in acute anterior poliơ myelitis an apparent defect of neuro-muscular transo mission can be detected by repetitive stimulation. 